\title{
Generic Patch Inference
}

Andersen, Jesper; Lawall, Julia Laetitia

Published in:

Automated Software engineering 2008

DOI:

10.1109/ASE.2008.44

Publication date:

2008

Document version

Publisher's PDF, also known as Version of record

Citation for published version (APA):

Andersen, J., \& Lawall, J. L. (2008). Generic Patch Inference. In Automated Software engineering 2008: 3rd IEEE/ACM International Conference on Automated Software Engineering (ASE 2008), September 15-19, 2008 (pp. 337-346). IEEE Communications Society. https://doi.org/10.1109/ASE.2008.44 


\section{Generic Patch Inference}

\author{
Jesper Andersen \\ DIKU, University of Copenhagen, \\ Universitetsparken 1, 2100 Copenhagen $\varnothing$, Denmark \\ Email: jespera@diku.dk
}

\author{
Julia L. Lawall \\ DIKU, University of Copenhagen, \\ Universitetsparken 1, 2100 Copenhagen $\varnothing$, Denmark \\ Email: julia@diku.dk
}

\begin{abstract}
A key issue in maintaining Linux device drivers is the need to update drivers in response to evolutions in Linux internal libraries. Currently, there is little tool support for performing and documenting such changes.

In this paper we present a tool, spdiff, that identifies common changes made in a set of pairs of files and their updated versions, and extracts a generic patch performing those changes. Library developers can use our tool to extract a generic patch based on the result of manually updating a few typical driver files, and then apply this generic patch to other drivers. Driver developers can use it to extract an abstract representation of the set of changes that others have made.

Our experiments on recent changes in Linux show that the inferred generic patches are more concise than the corresponding patches found in commits to the Linux source tree while being safe with respect to the changes performed in the provided pairs of driver files.
\end{abstract}

\section{INTRODUCTION}

In the case of open-source software, such as Linux, where the developers are widely distributed, it must be possible to exchange, distribute, and reason about source code changes. One common medium for such exchange is the patch [1]. When making a change in the source code, a developer makes a copy of the code, modifies this copy, and then uses diff to create a file describing the line-by-line differences between the original code and the new version. He then distributes this file, known as a patch, to subsystem maintainers and mailing lists for discussion. Once the patch has been approved, other developers can apply it to their own copy of the code, to update it to the new version.

Patches have been undeniably useful in the development of Linux and other open-source systems. However, it has been found that they are not ideal for one kind of change, the collateral evolution [2]. A collateral evolution is a change entailed by an evolution that affects the interface of a library, and comprises the modifications that are required to bring the library clients up to date with this evolution. Collateral evolutions range from simply replacing the name of a called library function to more complex changes that involve multiple parts of each affected file. Such changes may have to be replicated across an entire directory, subsystem implementation, or even across the entire source code. In the case of Linux, it has been shown that collateral evolutions particularly affect device drivers, where hundreds of files may depend on a single library [2].

The volume and repetitiveness of collateral evolutions strain the patch-based development model in two ways. First, the original developer has to make the changes in every file, which is tedious and error prone. Second, developers that need to read the resulting patch, either to check its correctness or to understand what it will do to their own code, may have to study hundreds of lines of patch code, which are typically all very similar, but which may contain some subtle differences. An alternative is provided by the transformation system Coccinelle, which raises the level of abstraction of patches to semantic patches [3]. A semantic patch describes a change at the source code level, like an ordinary patch, but is applied in terms of the syntactic and semantic structure of the source language, rather than on a line-by-line basis. Semantic patches include only the code relevant to the change, can be abstracted over irrelevant subterms using metavariables, and are independent of the spacing and line breaks of the code to which they are applied. The level of abstraction of semantic patches furthermore implies that they can be applied to files not known to the original developer, such as the many drivers that are maintained outside the Linux source tree.

Despite the many advantages of semantic patches, it may not be reasonable to expect developers to simply drop the patchbased development model when performing collateral evolutions. For the developer who makes the collateral evolution, there can be a gap between the details of an evolution within a library and the collateral evolution it entails. Therefore, he may still find it natural to make the required changes by hand in a few typical files, to better understand the range and scope of the collateral evolution that is required. Furthermore, the standard patch application process is very simple, involving only replacing one line by another, which may increase confidence in the result. Thus, developers may find it desirable to continue to distribute standard patches, with or without an associated semantic patch.

What is then needed is a means of mediating between standard patches and semantic patches, by inferring semantic patches from standard patches. In this paper, we propose a tool, spdiff, that infers a restricted form of semantic patch, which we refer to as a generic patch, from a collection of standard patches implementing a common set of transformations. The Linux developer who makes a change in a library that affects its interface can perform the collateral evolution in a few files based on his knowledge about how drivers typically make use of the library, and then apply spdiff to produce a generic patch that can be applied to the other files automatically. Complementarily, the developer who needs to read an existing 
standard patch implementing a collateral evolution can apply spdiff to the patch to obtain a more concise, abstract representation of the changes that are performed, as well as information about any deviations from these changes, which may represent bugs or special cases of which he should be aware. If the developer maintains proprietary code outside the Linux kernel source tree, he may furthermore use the inferred generic patch to apply the necessary changes.

Concretely, the contributions of this paper are:

- We provide a formalization of what constitutes a concise and abstract generic patch. The formalization does not rely on particular features of our generic patches and thus could be instantiated for other transformation languages.

- We give an algorithm that infers concise and abstract generic patches. We have implemented the algorithm speind in a tool spdiff.

- We show examples of the generic patches inferred by spdiff from some recent collateral evolutions in Linux.

The rest of this paper is organized as follows. Section II presents a motivating example that illustrates some of the issues taken into account by our approach. Sections III through $\mathrm{V}$ formally present our algorithm for inferring generic patches, by first defining a core term language, then developing the necessary elements of a theory of patches on this language, and finally defining the algorithm itself. Section VI illustrates the application of our spdiff tool to various recent collateral evolutions performed in the Linux source tree. Section VII describes related work and Section VIII concludes.

\section{Motivating EXAMPLE}

To motivate the design of spfind, we begin with a simple example of a collateral evolution from March $2007^{1}$ and consider the issues involved in inferring a generic patch for it. The collateral evolution required replacing uses of the general-purpose memory copying function memcpy that manages network buffers by calls to a special-purpose function, skb_copy_from_linear.

Figure 1 shows extracts of two files affected by this collateral evolution and the updates to these files. The lines prefixed with - and + indicate where code was removed and added, respectively. Furthermore, the line that is prefixed with ! has superficially the same form as the others, in that it represents a call to memcpy, but it is not affected by the collateral evolution. In the first file, two calls to memcpy are present and only one was affected and in the second file only one such call was affected.

A summary of the set of changes is shown in Figure 2. The summary reveals that although there are differences in how the two files were modified, there are also compelling similarities:

1) All calls to memcpy where the second argument is a reference to the field data are changed into calls

${ }^{1}$ Git SHA1 identification codes

$1 \mathrm{a} 4 \mathrm{e} 2 \mathrm{~d} 093 \mathrm{fd} 5 \mathrm{f} 3 \mathrm{eaf} 8 \mathrm{cffc} 04 \mathrm{a} 1 \mathrm{~b} 803 \mathrm{f} 8 \mathrm{~b} 0 \mathrm{ddef} 6 \mathrm{~d}$ and d626f62b11e00c16e81e4308ab93d3f13551812a.

All patches in this paper can be obtained from

http://git.kernel.org/?p=linux/kernel/git/torvalds/linux-2.6.git;a=summary

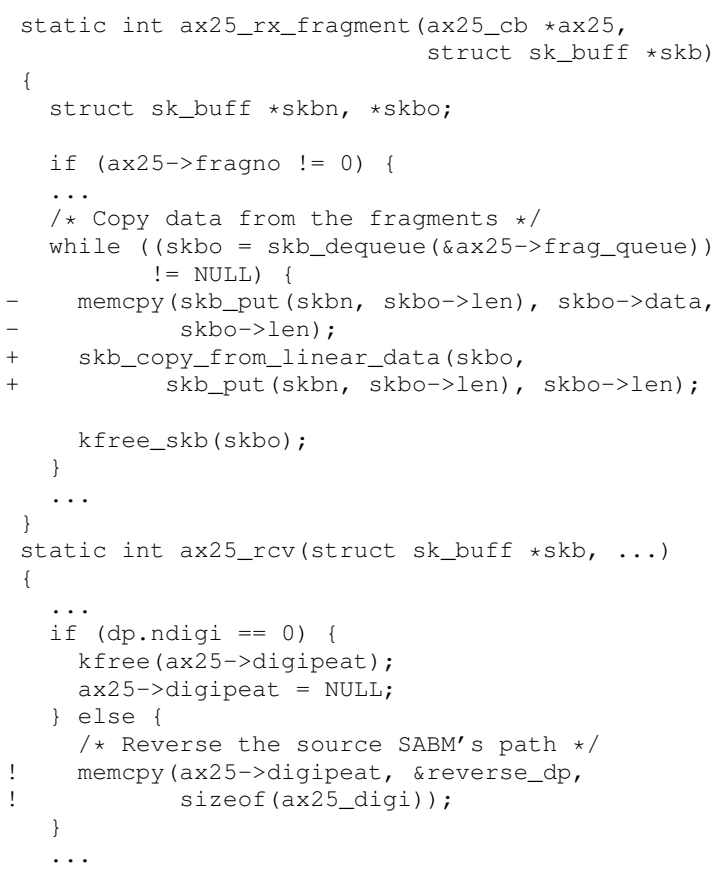

File: net/ax25/ax25_in.c

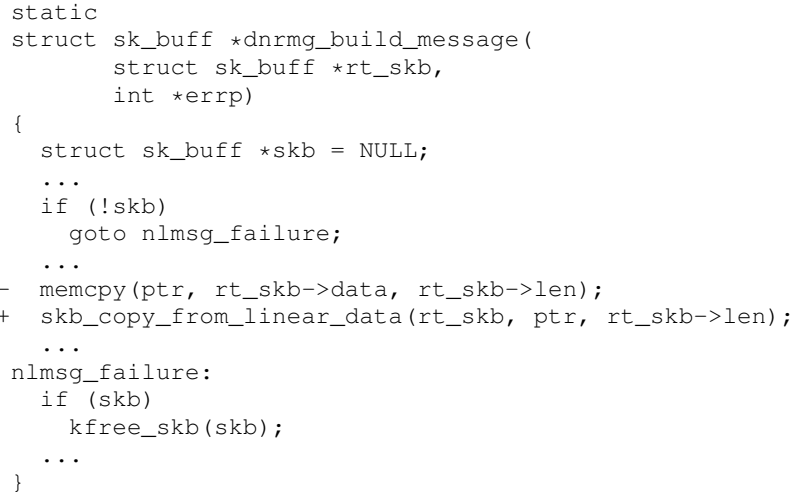

File: net/decnet/netfilter/dn_rtmsg.c

Fig. 1. Extracts of the two files.

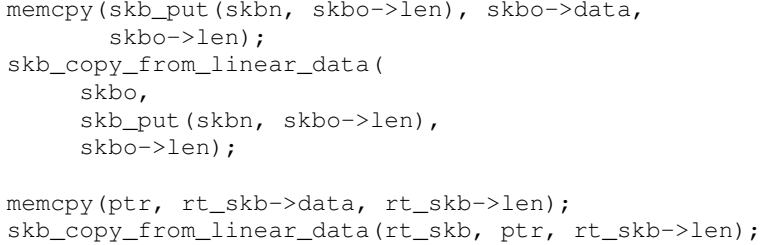

Fig. 2. Set of changes for the two files of Figure 1.

to skb_copy_from_linear_data. On the other hand, in the call to memcpy marked with a !, the second argument is not a reference to the field data.

2) The first argument becomes the second.

3) The field reference to data in the second argument is dropped. The resulting expression, which has type 
struct sk_buff $\star$, becomes the first argument of the new function call.

4) The third argument of memcpy is copied as-is to the third argument of the new function call.

The changes made to the two mentioned files can be summarised compactly as the following generic patch derived using our inference tool:

memcpy (X0, X1->data, X2)

skb_copy_from_linear_data (X1, x0, X2)

where $\mathrm{X} 0, \mathrm{X} 1$, and $\mathrm{X} 2$ serve as placeholders (metavariables) for concrete arguments. Intuitively, the above is an abstract representation of the changes made: in the context of a call to memcpy where the first and third argument are arbitrary expressions and the second references the data field, change the called function to skb_copy_from_linear_data, move the first argument to the second position, remove the data field reference of the second argument and make it the first, and copy the third argument as-is. Thus, the combined requirements on the context in which to make a transformation ensure that only the calls marked with - are affected and leave out the call to memcpy marked with !, as required.

As illustrated by this example, there are two main issues to be considered when inferring such generic patches: 1) compactness and 2) safety.

Compactness: The most trivial way to construct a generic patch is simply to enumerate the changes, as done for the example earlier in this section. The result, however, would be no better than a standard patch, and it would generally not be applicable to files other than the ones used for the inference. Finally, it would generally not be readable as high-level documentation of the changes performed. We prefer, therefore, a more compact description of the changes, which we produce by replacing subterms that are not affected by the transformation by metavariables, as illustrated by e.g., the use of $\mathrm{x} 0$ rather than the concrete terms skb_put (skbn, skbo->len) (in ax25_in.c) and ptr (in dn_rtmsg.c) in the generic patch in the above example.

Safety: The safety of a generic patch requires that only things that actually changed in the original file should be changed by the inferred generic patch. In our example, one of the calls to memcpy was not changed. We saw that we could ensure safety by imposing a structural restriction on the second argument to memcpy: only those calls where the second argument referenced the dat a field should be affected.

In the next two sections we develop the machinery needed in order to state an algorithm that can automatically infer safe and compact generic patches such as the one shown above. In the example, there was only one change, but the method we describe is capable of dealing with multiple changes, and always ensures that the derived generic patch correctly describes a transformation that applies to all the files given as input.

\section{SETUP}

While our approach targets $\mathrm{C}$ code, we formalise it in terms of a simpler language, which we call the language of TERMs. This language only distinguishes between atomic and compound terms, as is sufficient for the presentation of the algorithm. The language is defined as follows:

\section{Definition 1 (Syntax of Terms)}

$$
\text { TERM }::=\text { ATOM } \mid \operatorname{ATOM}\left(\text { TERM }^{+}\right)
$$

In this definition, and subsequently, $t^{+}$indicates one or more comma-separated occurrences of the nonterminal $t$. Furthermore, terms will be written as $a$ and $a(t s)$, for atomic and compound terms respectively.

Updates on terms are described by generic patches. A generic patch is created out of patterns, as defined below.

Patterns: A pattern is a TERM that may additionally contain metavariables, which are placeholders for concrete terms. The syntax of patterns is as follows:

\section{Definition 2 (Syntax of Patterns)}

$$
p::=\operatorname{ATOM}\left|\operatorname{ATOM}\left(p^{+}\right)\right| \operatorname{Meta} \mid \operatorname{Meta}\left(p^{+}\right)
$$

Where Meta denotes a set of metavariables. In the examples, we use uppercase letters to denote metavariables.

A pattern $p$ matches a term $t$ if there is a substitution $\theta$ of the metavariables for terms such that applying the substitution to the pattern yields a term syntactically equivalent to $t$, i.e., $\theta p=t$ where $\theta p$ denotes application of $\theta$ to $p$. A metavariable may occur more than once in a pattern, in which case all occurrences of the metavariable must match the same concrete term. For example, the pattern $f(X, X)$ matches any concrete term that is a call to $f$ with two syntactically equal arguments.

Term replacement patches: Patterns are then combined into term replacement patches. A term replacement patch describes how to transform any (sub)terms that match a given pattern. The syntax of a term replacement patch is as follows:

\section{Definition 3 (Syntax of Term Replacement Patches)}

$$
\operatorname{trp} \quad::=p \rightsquigarrow p
$$

The application of a term replacement patch to a term is defined by the rules shown in Figure $3 .^{2}$ Rule $a$ is concerned with the case where a term $t$ matches the pattern $p$ according to some substitution $\theta$. The matching term is replaced with $\theta p^{\prime}$. The remaining rules traverse the term top-down along all branches of the term (rule $b$ ) until reaching a subterm at which rule $a$ applies or until reaching a leaf (rule $c$ ). (Note that rules $b$ and $c$ only apply if rule $a$ does not.) If there is no matching subterm, the application of a term replacement patch behaves as the identity function.

\footnotetext{
${ }^{2}$ Note that although term replacement patches have the form of rewrite rules, they are not applied iteratively as done in term rewriting systems.
} 
(a)

$$
\begin{gathered}
\frac{\exists \theta: \theta p=t \quad \theta p^{\prime}=t^{\prime}}{\left(p \rightsquigarrow p^{\prime}\right)(t)=t^{\prime}, \top} \\
\left(p \rightsquigarrow p^{\prime}\right)\left(t_{i}\right)=t_{i}^{\prime}, f_{i} \text { for all } 0 \leq i \leq n \\
F=\bigsqcup f_{i} \\
\left(p \rightsquigarrow p^{\prime}\right)\left(a\left(t_{0}, \ldots, t_{n}\right)=a\left(t_{0}^{\prime}, \ldots, t_{n}^{\prime}\right), F\right. \\
\neg \exists \theta: \theta p=a \\
\frac{\left.\neg \rightsquigarrow p^{\prime}\right)(a)=a, \perp}{(p}
\end{gathered}
$$

Fig. 3. Application of a term replacement patch.

The application of a term replacement patch additionally returns a flag $T$, when a match has been found, or $\perp$, when no match has been found. These are ordered as $\perp \sqsubseteq T$. Note that a match may not actually cause the generated term to be different from the original one, e.g., if the term replacement patch specifies that the two arguments of a function should be switched, and they are actually textually equivalent.

Generic patches: A generic patch is a sequence of one or more term replacement patches, as defined by the following grammar:

Definition 4 (Syntax of Generic Patches) A generic patch is either a term replacement patch or a sequence of generic patches.

$$
\text { gp } \quad::=p \rightsquigarrow p \mid g p ; g p
$$

Subsequently, whenever we say "patch," we mean generic patch unless stated otherwise.

The rules for applying a generic patch are shown below. The application of a term replacement patch $p_{1} \rightsquigarrow p_{2}$ is defined in terms of the rules of Figure 3, but here the application only succeeds if the pattern matches somewhere, as indicated by the flag $T$. A sequence of patches $g p_{1} ; g p_{2}$ first applies $g p_{1}$ to the term and then the result of that application is used as input to the application of $g p_{2}$.

$$
\begin{array}{lll}
\llbracket p \rightsquigarrow p^{\prime} \rrbracket t & =t^{\prime \prime} & \text { if } p \rightsquigarrow p^{\prime}(t)=t^{\prime \prime}, \top \\
\llbracket g p_{1} ; g p_{2} \rrbracket t & =\llbracket g p_{2} \rrbracket\left(\llbracket g p_{1} \rrbracket t\right) &
\end{array}
$$

\section{THEORY OF SUBPATCHES}

To satisfy the criteria of safety and compactness, we would like to infer a generic patch that expresses the largest possible common transformation applied to each term, without performing any undesired transformations. In this section, we provide a formal definition of what it means for the transformation performed by a generic patch to be largest and common.

\section{A. Ensuring safety}

Safety of a generic patch requires that it does not perform undesired changes. Formally, safety is defined relative to a pair of terms $\left(t, t^{\prime \prime}\right)$ with the assumption that some transformation has been made in $t$ to turn it into $t^{\prime \prime}$. A patch $g p$ is safe relative to a pair of terms when the transformations it makes when applied to $t$ are a part of those that turn $t$ into $t^{\prime \prime}$. The following example shows a patch that performs a safe transformation and another patch that does not perform a safe transformation relative to a pair of terms.

Example 1 (Illustration of safety) Consider the following terms:

$$
\begin{aligned}
& t=m(f(10), f(100)) \\
& t^{\prime}=m(f(42), f(100)) \\
& t^{\prime \prime}=m(f(42), f(42)) \\
& t^{\prime \prime \prime}=m(f(42), f(420))
\end{aligned}
$$

and the following patches:

$$
\begin{aligned}
& g p^{\prime}=\mathrm{f}(10) \rightsquigarrow \mathrm{f}(42) \\
& g p^{\prime \prime}=\mathrm{f}(\mathrm{X}) \rightsquigarrow \mathrm{f}(42)
\end{aligned}
$$

Observe that $\llbracket g p^{\prime} \rrbracket t=t^{\prime}$ and $\llbracket g p^{\prime \prime} \rrbracket t=t^{\prime \prime}$. We now consider whether $g p^{\prime}$ or $g p^{\prime \prime}$ is safe with respect to the pair $\left(t, t^{\prime \prime \prime}\right)$.

The following diagram illustrates the application of $g p^{\prime}$ and $g p^{\prime \prime}$ to $t$, where subterms that have been affected by a patch are underlined, and the relation to the result, $t^{\prime \prime \prime}$ (center right).

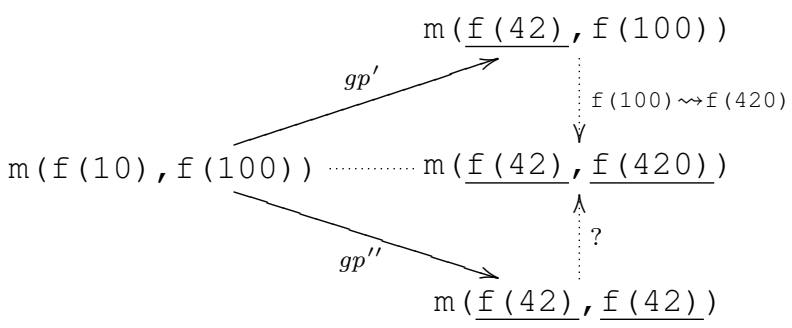

After the application of $g p^{\prime}$, only the subterm $f(100)$ (top right) of $t^{\prime}$ needs to be modified in order to reach $t^{\prime \prime \prime}$ (center right). As can be seen by the fact that $f(100)$ is not underlined it was not modified by $g p^{\prime}$. Thus, $g p^{\prime}$ is safe relative to $\left(t, t^{\prime \prime \prime}\right)$. After application of $g p^{\prime \prime}$, on the other hand, we need to undo some of the changes made by $g p^{\prime \prime}$, i.e., the second underlined occurrence of $\mathrm{f}(42)$ in $t^{\prime \prime}$ (bottom right). Thus, $g p^{\prime \prime}$ is not safe relative to $\left(t, t^{\prime \prime \prime}\right)$.

The definition of safety is based on the observation that in transforming $t$ into $t^{\prime \prime}$ it is not necessary to modify any subterm more than once. Thus, if $t^{\prime}$ is the result of applying $g p$ to $t$, then any subterm of $t$ that is changed in $t^{\prime}$ should not be changed again, and any subterm of $t$ that is not changed in $t^{\prime}$ can be changed in order to reach the third term $t^{\prime \prime \prime}$. The rules in Definition 5 capture this property. We then say that $g p$ is safe relative to $\left(t, t^{\prime \prime}\right)$.

Definition 5 (One-step reachable) Suppose a patch gp and a pair of terms $\left(t, t^{\prime \prime}\right)$ are given. Assume that $\llbracket g p \rrbracket t=t^{\prime}$ for some $t^{\prime}$. We say that $t^{\prime \prime}$ is one-step reachable if $t^{\prime \prime}$ can be obtained from $t^{\prime}$ without modifying any of the subterms that gp modified with respect to $t$. We denote this $t \rightarrow t^{\prime} \rightarrow t^{\prime \prime}$.

$$
\begin{gathered}
t=t^{\prime} \vee t^{\prime}=t^{\prime \prime} \\
t \rightarrow t^{\prime} \rightarrow t^{\prime \prime} \\
a(t s) \neq a^{\prime}\left(t s^{\prime}\right) \quad a^{\prime}\left(t s^{\prime}\right) \neq a^{\prime \prime}\left(t s^{\prime \prime}\right) \\
a=a^{\prime} \vee a^{\prime}=a^{\prime \prime} \\
\forall\left(t, t^{\prime}, t^{\prime \prime}\right) \in\left(t s, t s^{\prime}, t s^{\prime \prime}\right): t \rightarrow t^{\prime} \rightarrow t^{\prime \prime} \\
\hline a(t s) \rightarrow a^{\prime}\left(t s^{\prime}\right) \rightarrow a^{\prime \prime}\left(t s^{\prime \prime}\right)
\end{gathered}
$$


Based on Definition 5 we define a safe transformation part of a pair of terms $\left(t, t^{\prime \prime}\right)$ as a patch such that any affected subterm of $t$ is affected in a safe manner.

\section{Definition 6 (Safe Transformation part)}

$$
g p \preceq\left(t, t^{\prime \prime}\right) \Longleftrightarrow \forall t^{\prime}: \llbracket g p \rrbracket t=t^{\prime} \Rightarrow t \rightarrow t^{\prime} \rightarrow t^{\prime \prime}
$$

Common patch: A patch is said to be common to a set of pairs of terms $C$ if it is safe relative to each of the pairs of C.

Definition 7 (Common Safe Patch) A patch gp is considered common relative to a set of pairs of terms $C$ if it is safe relative to each pair of terms in the set $C$ :

$$
g p \preceq C \Longleftrightarrow \forall\left(t, t^{\prime \prime}\right) \in C: g p \preceq\left(t, t^{\prime \prime}\right)
$$

\section{B. Ensuring compactness}

In addition to seeking a safe generic patch, we also seek a generic patch that compactly represents the changes made. In order to define that a generic patch $g p^{\prime}$ is more compact than another generic patch $g p$, we define an ordering of patches relative to a pair of terms: $g p \preceq_{\left(t, t^{\prime \prime}\right)} g p^{\prime}$. When $g p \preceq_{\left(t, t^{\prime \prime}\right)} g p^{\prime}$ the transformations expressed by $g p$ are also contained in $g p^{\prime}$ and we say that $g p$ is a subpatch of $g p^{\prime}$.

\section{Definition 8 (Patch Ordering Relation)}

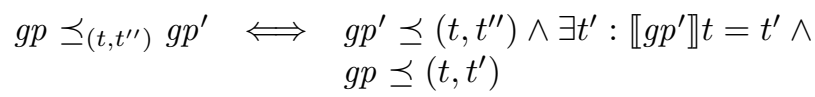

Thus, we consider $g p$ a subpatch of $g p^{\prime}$ if and only if $g p$ performs a safe part of the transformation that $g p^{\prime}$ performs, as expressed by $g p \preceq\left(t, t^{\prime}\right)$, and $g p^{\prime}$ is a safe part of $\left(t, t^{\prime \prime}\right)$.

The subpatch definition can be generalised to a set of pairs of terms as follows:

$$
g p \preceq_{C} g p^{\prime} \Longleftrightarrow \forall\left(t, t^{\prime \prime}\right) \in C: g p \preceq_{\left(t, t^{\prime \prime}\right)} g p^{\prime}
$$

Example 2 (Compactness and subpatches) In this example we show that 1) when a patch $g p^{\prime}$ is a larger than $g p$ it can actually be syntactically smaller than $g p$ and that 2) larger patches can be more compact by covering the changes expressed by several smaller patches.

Consider the following terms:

$$
\begin{aligned}
t_{f} & =\mathrm{f}(1) \\
t_{g} & =\mathrm{g}(\mathrm{f}(1)) \\
t_{h} & =\mathrm{h}(\mathrm{f}(1)) \\
t_{x} & =\mathrm{x}(\mathrm{f}(2)) \\
t_{f}^{\prime} & =\mathrm{f}(1,1) \\
t_{g}^{\prime} & =\mathrm{g}(\mathrm{f}(1,1)) \\
t_{h}^{\prime} & =\mathrm{h}(\mathrm{f}(1,1)) \\
t_{x}^{\prime} & =\mathrm{x}(\mathrm{f}(2,2)) \\
t_{m} & =\mathrm{m}(\mathrm{g}(\mathrm{f}(1)), \mathrm{h}(\mathrm{f}(1))+\mathrm{x}(2)) \\
t_{m}^{\prime} & =\mathrm{m}(\mathrm{g}(\mathrm{f}(1,1)), \mathrm{h}(\mathrm{f}(1,1)+\mathrm{x}(2,2)))
\end{aligned}
$$

as well as the following patches:

$$
\begin{aligned}
& g p_{f}=t_{f} \rightsquigarrow t_{f}^{\prime}=\mathrm{f}(1) \rightsquigarrow \mathrm{f}(1,1) \\
& g p_{g}=t_{g} \rightsquigarrow t_{g}^{\prime}=\mathrm{g}(\mathrm{f}(1)) \rightsquigarrow \mathrm{g}(\mathrm{f}(1,1)) \\
& g p_{h}=t_{h} \rightsquigarrow t_{h}^{\prime}=\mathrm{h}(\mathrm{f}(1)) \rightsquigarrow \mathrm{h}(\mathrm{f}(1,1)) \\
& g p_{x}=t_{x} \rightsquigarrow t_{x}^{\prime}=\mathrm{X}(\mathrm{f}(2)) \rightsquigarrow \mathrm{X}(\mathrm{f}(2,2)) \\
& g p_{f, x}=\mathrm{f}(\mathrm{X}) \rightsquigarrow \mathrm{f}(\mathrm{X}, \mathrm{X})
\end{aligned}
$$

The subpatch hierarchy is given below. An arrow from $g p_{i}$ to $g p_{j}$ indicates that relative to the term pair $\left(t_{m}, t_{m}^{\prime}\right), g p_{i}$ is a subpatch of $g p_{j}$.

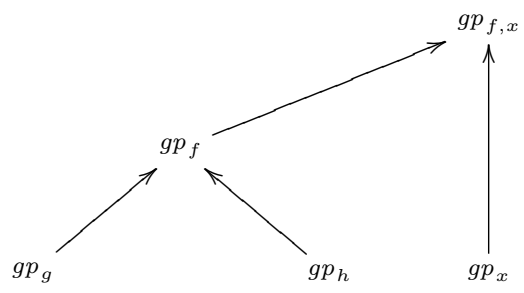

From the hierarchy it is evident that although $g p_{f}$ is syntactically smaller than both $g p_{g}$ and $g p_{h}$ it is a superpatch of both. Finally, we see that $g p_{f, x}$ is the largest patch and that it covers both the transformations made by $g p_{f}$ and $g p_{x}$. Therefore it is more compact than the combination of the two smaller patches.

The largest common subpatch: Suppose $C$ is a set of pairs of terms $\left\{\left(t_{0}, t_{0}^{\prime \prime}\right), \ldots,\left(t_{n}, t_{n}^{\prime \prime}\right)\right\}$ representing the original and updated code after some manual collateral evolution has been performed. A largest common subpatch for $C$ is then a patch $g p$ satisfying the following properties:

$$
\begin{array}{r}
g p \preceq C \\
\forall g p^{\prime}: g p^{\prime} \preceq C \Rightarrow g p^{\prime} \preceq_{C} g p
\end{array}
$$

Property (1) expresses that $g p$ is a common safe patch and property (2) expresses that $g p$ is largest among the common subpatches. Since there can be more than one patch satisfying the requirements above, we let $L C P(C)$ be the set of largest common patches for a set of pairs of terms $C$. The set $L C P(C)$ is thus the set of the most compact and safe generic patches relative to a set $C$.

Example 3 (Largest Common Subpatch) The following is an example of a largest common subpatch for a set of pairs of terms $\left\{\left(t_{1}, t_{1}^{\prime}\right),\left(t_{2}, t_{2}^{\prime}\right)\right\}$. This example shows that the largest common subpatch need not be unique.

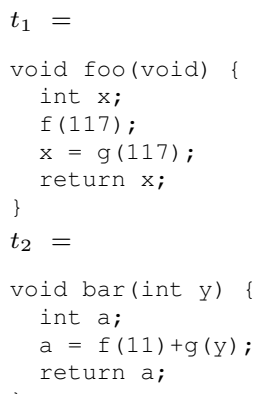

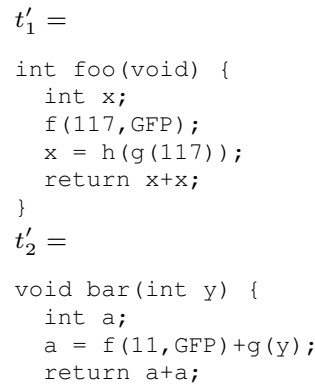


To generate all the concrete parts for a pair of terms $\left(t, t^{\prime \prime}\right)$ the algorithm simply traverses the two terms in parallel. For each pair of subterms it returns the concrete part $t_{l} \rightsquigarrow t_{r}$ only when $t_{l} \rightsquigarrow t_{r} \preceq\left(t, t^{\prime \prime}\right)$, using Definition 6 to perform the part-of check.

For each concrete part $t_{l} \rightsquigarrow t_{r}$, the algorithm then generates a set of generalised patches by introducing metavariables for subterms of $t_{l}$ and $t_{r}$ respectively such that $\exists \theta: \theta p=t_{l}$ and $\theta p^{\prime}=t_{r}$ and $p \rightsquigarrow p^{\prime} \preceq\left(t, t^{\prime \prime}\right)$. The pseudocode for introducing metavariables is tedious but straightforward and consequently not shown. As an example, however, consider the concrete term replacement patch $\mathrm{f}(42,117) \rightsquigarrow f(42,117+42)$. Below we show a non exhaustive list of possible abstracted versions:



Intersection: The final step of simple_pairs is to take the intersection of all the generated sets of term replacement patches. Because the generalization process ensures that all metavariables have been generated systematically, if I is the intersection of all sets of term replacement patches generated as described above, then it holds that:

$$
\forall g p \in I: g p \preceq \mathrm{C}
$$

where $C$ is the set of pairs of terms input to the simple_pairs function. Recall that the definition of $g p \preceq$ C simply requires that $g p$ is a safe transformation part for each pair in C. Since I is the intersection of all the sets of term replacement patches constructed from each pair of terms in $\mathrm{C}$ ( $\mathrm{B}_{i}$ in line 6 ), we can conclude that $\forall\left(t, t^{\prime}\right) \in \mathrm{C}: g p \preceq\left(t, t^{\prime}\right)$ as required.

\section{B. Creating sequential patches}

The gen function (lines 8 to 19 in Figure 4) constructs larger patches based on the term replacement patches returned by the simple_pairs function.

A technically correct version of the gen function would simply generate all possible permutations of the term replacement patches given, and then extract the largest among the constructed patches. This approach, however, is unnecessarily inefficient. Instead, the algorithm iteratively constructs larger patches by extending simple patches into sequential ones and removing smaller ones when combining sets of results. To remove smaller patches, the algorithm uses Definition 8 for $g p \preceq C g p^{\prime}$.

Finding possible next patches: Given a set of pairs of terms, a prefix patch (denoted current_bp in Figure 4) and a set of term replacement patches, the function next_bps returns the set of patches that can safely be applied after current_bp. For each of these possible next patches (denoted bp), the algorithm extends current_bp into current_bp; bp (in line 15) and recursively calls gen with the extended patch. If there are no more next patches to apply, the algorithm simply returns the singleton set consisting of the current prefix patch. The function next_bps is defined as:

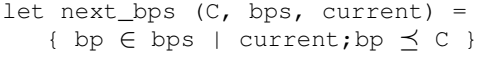

Joining sets of patches: Given sets of constructed patches, lines 18-19 combine them to obtain a resulting set that has the property that all patches in the set are largest:

$$
\mathrm{S} * \mathrm{~S}^{\prime}=\left\{g p \in S \cup S^{\prime} \mid \forall g p^{\prime} \in S \cup S^{\prime}: g p^{\prime} \preceq_{C} g p\right\}
$$

Correctness of algorithm: We now state the relation between the set of largest common subpatches $L C P(C)$ and the algorithm, although a formal proof of correctness is beyond the scope of this paper.

The algorithm given in Figure 4 is sound and complete with respect to the definition of set of largest common patches. Given a set of pairs of terms $C$, spfind $(C) \subseteq L C P(C)$ and $L C P(C) \subseteq \operatorname{spfind}(C)$.

The implementation: We have implemented the algorithm in a tool called spdiff. ${ }^{3}$ Because $\operatorname{LCP}(\mathrm{C})$ can be large and involve uninteresting or overly generic variants, we have desiged the tool to be sound but not complete, although the tool always produces non-empty results when $L C P(C) \neq \emptyset$. In the following we consider in more detail what these uninteresting or overly generic variants may be.

In Example 3 we have already seen a case where $L C P(C)$ contains multiple elements because of differences in the order of the constituent term replacement patches. $L C P(C)$ can also be very large when there are too many opportunities for generalization. Given a set $C$ of pairs of terms, the set $L C P(C)$ may be very large in case the terms in $C$ are structurally very similar. To see why, suppose the following generic patch is in the set $L C P(C)$ :

$$
\begin{aligned}
& \text { foo }(\operatorname{bar}(w+x), 117+y) \\
& \rightsquigarrow \text { foo }(\operatorname{bar}(w+x), 117+y)+z
\end{aligned}
$$

The essential part of the change is that in the right hand side, a $\mathrm{z}$ is added. Potentially, however any subexpression can be abstracted, so there are $O\left(n^{2}\right)$ abstractions, $g p$, of the generic patch such that $g p \in L C P(C)$. Given that the above generic patch is in the set $L C P(C)$, it may be that the following generic patches are also in this set (recall that metavariables are written as uppercase letters).

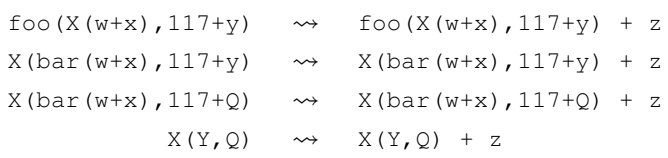

Although $\mathrm{X}(\mathrm{Y}, \mathrm{Q}) \rightsquigarrow \mathrm{X}(\mathrm{Y}, \mathrm{Q})+\mathrm{z}$ is in the set $L C P(C)$ it is not very likely that this generic patch is going to be safe to apply to an unknown input term, because it specifies no concrete information about either the function that is applied or its arguments. Thus, the tool discards such a result, if a more informative result is available.

\footnotetext{
${ }^{3}$ Binary available as http://www.diku.dk/ $\sim$ jespera/spdiff.opt
} 
Based on the observation expressed in Theorem 1 that all generic patches in the set $L C P(C)$ perform the same transformation to all given terms, the tool can return a subset $L C P(C)$ of generic patches that seem likely to also apply correctly to unknown terms. Concretely, define the size of a generic patch $|g p|$ as the size of each pattern it contains, where the size of a pattern is given by:

$$
\begin{array}{ll}
|\operatorname{ATOM}| & =1 \\
\left|\operatorname{ATOM}\left(t_{0}, \ldots, t_{n}\right)\right| & =1+\left|t_{0}\right|+\cdots+\left|t_{n}\right| \\
|\operatorname{Meta}| & =0 \\
\left|\operatorname{Meta}\left(t_{0}, \ldots, t_{n}\right)\right| & =\left|t_{0}\right|+\cdots+\left|t_{n}\right|
\end{array}
$$

The size of a pattern is thus the number of ATOMs in the pattern. Let spdiff $(C)$ denote the result of running the tool with input $C$. The relation between the result of spdiff $(C)$ and $L C P(C)$ is then

$$
\begin{aligned}
& \text { spdiff }(C)=R \wedge L C P(C)=B \Rightarrow \\
& R \subseteq B \wedge \\
& \quad R=\left\{g p\left|g p \in B, \forall g p^{\prime} \in B:\right| g p^{\prime}|\leq| g p \mid\right\}
\end{aligned}
$$

The size of the previous generic patch is then:

$$
\begin{aligned}
& |\mathrm{X}(\mathrm{Y}, \mathrm{Q}) \rightsquigarrow \mathrm{X}(\mathrm{Y}, \mathrm{Q})+\mathrm{Z}|= \\
& \quad|\mathrm{X}(\mathrm{Y}, \mathrm{Z})|+|\mathrm{X}(\mathrm{Y}, \mathrm{Z})+\mathrm{Z}|=0+1=1
\end{aligned}
$$

The other generic patches listed above have a size that is strictly larger than 1 . Thus, the above generic patch is not part of the set returned by the tool.

\section{EXAMPLES}

We now provide a few examples of the use of spdiff, based on some recent patches committed to Linux that we have identified using the patchparse collateral evolution mining tool [2]. For each standard patch that we have tested, we have constructed the set of pairs of terms, $C$, from the image of the Linux source tree just before the standard patch was applied and just after.

Adapt to structure changes: The following commits, dated November 9, 2007, begin with the log message "convert to use the new SPROM structure". ${ }^{4}$

95de2841aad971867851b59c0c5253ecc2e19832

$458414 \mathrm{~b} 2 \mathrm{e} 3 \mathrm{~d} 9 \mathrm{dd} 7 \mathrm{ee} 4510 \mathrm{~d} 18 \mathrm{c} 119 \mathrm{a} 7 \mathrm{ccd} 3 \mathrm{~b} 43 \mathrm{ec} 5$

7797 aa384870e3bb5bfd3b6a0eae61e7c7a4c993

These commits comprise over 650 lines of patch code, and affect 12 files in the drivers/net directory or its subdirectories, at 96 locations. In the role of an expert in the affected files, we selected three files from the first commit that illustrate the set of required changes. From these files, spdiff infers the following generic patch:

X0->sprom.r1 $\rightsquigarrow \mathrm{X} 0->$ sprom ;

sprom->r1.X0 $\rightsquigarrow$ sprom $->X 0$

The inferred generic patch fully updates all 12 original files in the same way the standard patches did. By careful examination a person could construct the inferred generic patch by hand. However, there would be no guarantee that the constructed

\footnotetext{
${ }^{4}$ The patches can be obtained from

http://git.kernel.org/?p=linux/kernel/git/torvalds/linux-2.6.git;a=summary
}

patch is safe, as this is not evident in the standard patch. To check safeness manually, one would have to consider 1) whether the constructed patch updates the proper locations correctly but does not update locations, that were not to be modified, and 2) whether the constructed patch is only a part of the update that is to be performed to a particular file.

Furthermore, the inferred generic patch updates some other files that were present at the time of the original patches but were overlooked. These files were in other directories and were not updated until February 18, 2008, by another developer.

Structure changes: Commit c32c2f63a9d6c953aaf168c0b2551da9734f76d2 from February 14, 2008 affects 9 files at 12 locations. The message attached to the commit is "d_path: Make seq_path () use a struct path argument". The standard patch attached to the commit is approximately 160 lines. The patch inferred by spdiff is:

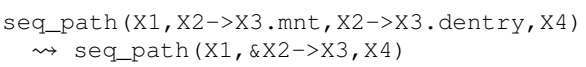

The inferred generic patch fully updates all but one of the original files. The only file that is not fully updated is the file fs/namespace.c in which a declaration struct path mnt_path is also added.

Renaming of function calls: The following commits, dated December 20, 2007, begin with some variant of the log message "Kobject: convert drivers/* from kobject_unregister() to kobject_put ()".

c10997f6575f476ff38442fa18fd4a0d80345f9d

$78 \mathrm{a} 2 \mathrm{~d} 906 \mathrm{~b} 40$ fe 530 ea $800 \mathrm{c} 1 \mathrm{e} 873 \mathrm{bfe} 8 \mathrm{f} 02326 \mathrm{f} 1 \mathrm{e}$

197b12d6796a3bca187f22a8978a33d51e2bcd79

38 a 382 ae 5 dd 4 f 4 d0 4 e 3046816 b0a 41836094 e 538

These commits comprise almost 800 lines of patch code, and affect 35 files at 79 locations. Based on the changes in the 17 files in the first of the above commits, spdiff derives the following generic patch:

kobject_unregister (X0) kobject_put (X0)

The inferred generic patch fully updates all but 3 files in the same way the standard patch did. The remaining files each include an additional change that goes beyond the collateral evolution.

Modifying declarations: Commit c11ca97ee9d2ed593ab7b5523def7787b46f398f and 12 others from around December 7, 2007 change 21 files at 26 locations. The log messages are "use LIST_HEAD instead of LIST_HEAD_INIT". The standard patches total almost 300 lines. The inferred generic patch is:



The inferred generic patch fully updates all 21 files. The original developer, on the other hand, initially overlooked one case and had to create a second patch on the same file to correct it. Furthermore, 6 files that contained relevant declarations at the time the patches were committed were not updated by the original patches, and of those 5 files have still not been updated today (four months later). All of these files are fully updated by the generic patch. 
Use kzalloc: Over the past couple of years, around 100 patches have been committed that convert the combination of calls to kmalloc and memset to kzalloc. One such commit is dd3927105b6f65afb7dac17682172 cdfb8$6 \mathrm{~d} 3 \mathrm{f} 00$ from September 6, 2005 which affected 6 files at 27 locations. The transformation it performs can be represented as follows.

$$
\left.\begin{array}{l}
x=\operatorname{kmalloc}(\text { size, flags) } ; \\
\ldots \\
\operatorname{memset}(x, 0, \text { size) } ;
\end{array}\right\} \rightsquigarrow \begin{aligned}
& \operatorname{kzalloc}(x, \text { size, flags) } \\
& \ldots
\end{aligned}
$$

Our tool is, however, not able to infer any common generic patch in this case because the language of generic patches is not able to express the temporal ordering of terms or the sharing of metavariables between disjoint code fragments.

Assessment: These examples show that for a variety of collateral evolutions, spdiff infers generic patches that are much more concise, and we believe much more readable, than the corresponding standard patches. In several cases, the original standard patches did not perform part of the collateral evolution in some relevant files. In this situation, a developer could use spdiff to infer a generic patch from the provided standard patches and to complete the collateral evolution. Using the Coccinelle transformation system, the generic patch can be applied everywhere in the Linux source tree.

While all of the inferred generic patches inferred are all simple enough that a person could construct them by hand by inspecting the standard patches, it would require more work to confirm that the manually constructed patch is indeed safe for all of the input files. Safeness is not evident from the standard patches, so to confirm safeness, one would need to apply the constructed patch to all original input files and check that for each file, the constructed patch applies correctly to a subset of the locations that need to be modified in the file.

Our final example illustrates a limitation of generic patches. The richer language of semantic patches provided by Coccinelle can express the properties needed to treat such examples [3]. We plan to extend spdiff to treat such cases in the future.

\section{RELATED WORK}

Our approach considers the problem of finding a single generic patch that correctly updates a collection of programs. We know of no work that addresses this problem directly. Several approaches, however, have considered how to concisely capture the changes between the original and modified versions of a single program. In this section we relate our approach to a number of other approaches that detect programs changes.

Chawathe et al. [4] describe a method to detect changes to structured information based on an ordered tree and its updated version. Their goal is to derive a compact description of the changes. To this end, a notion of a minimum cost edit script is defined. An edit script is basically a sequence of operations where each operation has an associated cost determined by some measure of structural similarities between the trees.
As such, the minimum cost edit script will be the most compact description of the changes made to the original tree with respect to the edit operations. Edit operations, however, always explicitly denote the node to transform and thus the approach is not sufficient for our context where we would like one transformation specification that applies to even unknown code.

Neamtiu et al. consider the problem of identifying changes to $\mathrm{C}$ programs [5]. Their method infers changes, additions and deletions of various program elements based on structure matching of syntax trees. Two trees that are structurally identical but have differences in their nodes are considered to represent matching program fragments. In contrast to the work by Chawathe et al., each simple change (e.g. renaming of a variable) is only reported once. Thus, the description of the changes made can be more compact than what is possible with the minimum cost edit scripts of Chawathe et al. However, similarities in changes involving larger trees are not detected, and consequently very similar changes made across all functions are reported as separate changes, whereas we need to be able to generalise descriptions of changes.

Kim et al. propose a method to infer "change-rules" from two versions of the same program [6]. Their goal is to construct a small set of change rules that capture many changes. Change rules express changes related to program headers (method headers, class names, package names, etc.). The basic shape of a change rule is similar to that of our term replacement patches: $\forall x \in$ scope : transformation, meaning that every match described by the scope is modified by the transformation. The scope, described using a variant of regular expressions, ranges over the textual representations of the previously mentioned headers. By using regular expressions as an abstraction mechanism the scope can be extended to e.g. all calls to a method that starts with the prefix foo. Thus, change rules can express that a given transformation was applied to a set of entities which is more compact than simply enumerating all entities. Our term replacement patches are similar to change rules but apply to any program element rather than just to program headers. Finally, our use of metavariables allow equality constraints among program elements as well as applicability to more than one input program.

Weißgerber et al. present a technique to identify likely refactorings in the changes that have been performed in Java programs [7]. Like Kim et al., they search for a fixed set of transformation types (in this case, rename method, add parameter, etc). Each transformation type has an associated precondition that enables the transformation. They first collect various signature information about the old and new versions of a given file, and then use this information to determine whether the preconditions of any of the transformation types is satisfied. If a precondition is satisfied, the transformation is considered a refactoring candidate. They furthermore use clone-detection to check that the change performed by a candidate is semantics preserving. Because we consider arbitrary changes, such checks are not relevant in our case. The transformation types given by Weißgerber et al. do not support 
any kind of abstraction mechanisms such as our metavariables. Thus, two detected changes can not be generalised into a more compact description that covers both of them, as could potentially be done by the method given by Kim et al. and by our work.

The patchparse collateral evolution mining tool [2] scans patch files for frequently occurring changes, modulo a simple strategy for abstracting away from terms that are shared between the original and modified code. Patchparse is sufficient to detect some of the rules in our examples, such as the kobject_unregister/kobject_put example, for which it reports:

kobject_unregister(ARG0) replaced by kobject_put (ARG0)

However, its strategy for detecting common terms is essentially top-down, and thus it reports the following result for the seq_path example:

seq_path (ARG0, CODE, CODE, ARG3) replaced by

seq_path (ARG0, CODE, ARG3)

In this case, it was not able to relate the second and third arguments in the original call to the second argument in the new code, and thus it falls back on characterizing these arguments as arbitrary code (CODE), which is not sufficient to specify the transformation. Patchparse furthermore does not ensure that the transformation represented by the proposed collateral evolution is either safe or compact. It can, however, be beneficially used, as we have done in this paper, to narrow down the set of patches considered when using spfind to infer generic patches based on standard patches already submitted to Linux.

\section{CONCLUSION}

The contributions of this paper are as follows: 1) We provide a formalisation of the largest common subpatch notion independent of the transformation language used. 2) We give an algorithm that performs inference of largest common subpatches relative to a transformation language of generic patches as well as a tool that implements the algorithm. 3) We have shown examples of inferred generic patches from recent collateral evolutions in Linux where our tool infers generic patches that are more compact than the standard patches that were originally applied to the Linux source tree and allowed us to update relevant files where the collateral evolution was not performed.

Currently, the speind tool requires that a change be made in all of the provided files. In practice, however, particularly when spfind is used to better understand a collection of existing standard patches, it can be useful to be able to detect changes that occur in only a subset of the files. We plan to address this issue in the near future, e.g., by allowing the user to provide a threshold for the frequency of occurrences required for a change to be considered for inclusion in the generic patch.

Finally, the language of generic patches can cover only a subset of the collateral evolutions performed in the Linux kernel. In the future we plan to extend our method to the richer language of semantic patches provided by Coccinelle.

\section{ACKNOWLEDGMENTS}

We would like to thank Gilles Muller for his feedback on an earlier version of this paper. We would also like to thank the anonymous reviewers for helpful comments. And finally, this work was supported in part by the Danish Research Council for Technology and Production Sciences.

\section{REFERENCES}

[1] D. MacKenzie, P. Eggert, and R. Stallman, Comparing and Merging Files With Gnu Diff and Patch. Network Theory Ltd, Jan. 2003, unified Format section, http://www.gnu.org/software/diffutils/ manual/html_node/Unified-Format.html.

[2] Y. Padioleau, J. L. Lawall, and G. Muller, "Understanding collateral evolution in Linux device drivers," in The first ACM SIGOPS EuroSys conference (EuroSys 2006), Leuven, Belgium, Apr. 2006, pp. 59-71.

[3] Y. Padioleau, J. Lawall, R. R. Hansen, and G. Muller, "Documenting and automating collateral evolutions in Linux device drivers," in Eurosys 2008, Glasgow, Scotland, Mar. 2008, pp. 247-260.

[4] S. S. Chawathe, A. Rajaraman, H. Garcia-Molina, and J. Widom, "Change detection in hierarchically structured information," in SIGMOD '96: Proceedings of the 1996 ACM SIGMOD international conference on Management of data. New York, NY, USA: ACM, 1996, pp. 493-504.

[5] I. Neamtiu, J. S. Foster, and M. Hicks, "Understanding source code evolution using abstract syntax tree matching," SIGSOFT Softw. Eng. Notes, vol. 30, no. 4, pp. 1-5, 2005

[6] M. Kim, D. Notkin, and D. Grossman, "Automatic inference of structural changes for matching across program versions," in ICSE '07: Proceedings of the 29th International Conference on Software Engineering. Washington, DC, USA: IEEE Computer Society, 2007, pp. 333-343.

[7] P. Weissgerber and S. Diehl, "Identifying refactorings from source-code changes," in ASE '06: Proceedings of the 21st IEEE/ACM International Conference on Automated Software Engineering. Washington, DC, USA IEEE Computer Society, 2006, pp. 231-240. 